\title{
ERP evidence for scarce rule representation in older adults following short, but not long preparatory intervals
}

\author{
Daniela Czernochowski* \\ Department of Experimental Psychology, Heinrich-Heine University Düsseldorf, Düsseldorf, Germany
}

\section{Edited by:}

Shulan Hsieh, National Cheng Kung

University, Taiwan

Reviewed by:

Andreas Keil, University of Florida, USA

Yoav Kessler, University of Toronto, Canada

\section{*Correspondence:}

Daniela Czernochowski, Department of Experimental Psychology,

Heinrich-Heine University Düsseldorf, Universitätsstr. 1, Gebäude,

23.03.U1.82, Düsseldorf, Germany. e-mail: d.czernochowski@hhu.de
The focus of the present study was to examine the cognitive processes comprising advance preparation - rule representation, task-set updating, and task-set reconfiguration - in young (20-25 years) and older adults (61-83 years). Specifically, this study aimed at further characterizing age-related differences in advance preparation, and evaluating how additional time to prepare might reduce behavioral costs in older adults. In line with previous findings, reaction time mixing costs were slightly larger for older compared to young adults, whereas behavioral switch costs were age-invariant. Following short preparation $(600 \mathrm{~ms})$, smaller antero-frontal event-related potential (ERP) correlates of rule representation were associated with pronounced congruency costs in older adults. Centro-parietal ERP correlates of task-set updating and task-set reconfiguration were not delayed, but smaller in magnitude for older compared to young adults. Longer preparation (1200 ms) enabled older adults to re-activate relevant task rules, as evident in reduced congruency costs, and temporally sustained ERP correlates of task-set updating and rule representation well beyond $600 \mathrm{~ms}$. Age-invariant switch costs appear related to additional, potentially compensatory frontal activity recruited by older adults to overcome difficulties in task-set reconfiguration.

Keywords: executive functions, ERPs, aging, task-switch

\section{INTRODUCTION}

Everyday, behavior needs to be flexibly adapted in many different situations. In these instances, cognitive control is necessary to monitor changes in task demands and to select appropriate activities according to current priorities, in short, to coordinate goal-directed behavior (Koechlin et al., 2003). These functions have been ascribed to the prefrontal cortex (PFC), which exerts top-down control on more posterior brain regions to facilitate subsequent task-appropriate processing. According to the guided activation theory (cf. Miller and Cohen, 2001; O'Reilly et al., 2002), a balance between maintaining stable task representations and choosing appropriate degrees of flexibility is achieved through sustained activations in the PFC. These sustained PFC activations allow for rapid updating when task demands change, for instance in a task-switching paradigm.

While switching between several tasks might not be very difficult for most young people, older adults appear to be more vulnerable to such interruptions in ongoing task performance (e.g., Kray and Lindenberger, 2000). However, previous behavioral investigations (e.g., Cepeda et al., 2001; Meiran et al., 2001) have provided evidence that these age-related problems in cognitive control can be ameliorated when additional time is given to prepare for an upcoming task, for instance by presenting an informative cue some time before the target. Hence, the focus of the present study was to examine the cognitive processes comprising advance preparation in young and older adults and to determine the mechanisms responsible for older adults' behavioral benefits during task-switching following long compared to short cue-target intervals (CTIs).
Various experimental paradigms have been developed to examine the cognitive processes necessary to cope with frequent changes in task requirements. In the cued task-switching paradigm, participants are asked to complete either one block of the same task (i.e., homogeneous trials), or to change between two or more tasks after a certain number of trials in mixed blocks. To illustrate, in the paradigm employed here, participants were asked to categorize one of four stimuli (“1,”“111,”“3,” or “333”) based on which digit (task 1) or how many digits (task 2) were displayed. Importantly, the cue in homogeneous blocks carries very little task-relevant information because the task remains the same throughout the block. By contrast, the cue in mixed blocks informs participants whether the next target requires a response according to the task rule that was used on the previous trial (no-switch or stay trials) or a switch to the second task (switch trials). Hence, even before the presentation of the target, in mixed blocks the cue is categorized according to its current task relevance (no-switch or switch), which initiates other executive processes in preparation for the upcoming response.

The executive processes involved in task-switching have been divided into two broad categories, based on two types of behavioral costs reflected in longer reaction times (RTs) and/or decreased accuracy. Mixing costs are calculated as the difference between no-switch trials from mixed blocks compared to trials from homogeneous blocks and are thought to reflect the coordination of two (or more) task-sets (cf. Monsell, 2003). These task-set coordination processes include task-set updating to determine which task-set is currently relevant. Following the classification of the cue, no-switch trials also require the continued active maintenance of the previously relevant stimulus-response rule (i.e., rule 
representation, De Jong et al., 1999; Bunge, 2004; Crone et al., 2006b; Lenartowicz et al., 2010). By contrast, behavioral switch costs reflect the difference between switch and no-switch trials in mixed blocks. Because switch trials require the use of the alternate task-set's procedural rules, a number of different executive processes are thought necessary. These have been summarized under the term task-set reconfiguration. These processes include switching the attentional focus to the newly relevant task-set, the retrieval and implementation of the newly relevant task-set's rules, and the inhibition of the previously relevant taskset (cf. Monsell, 2003). Note that other mechanisms (e.g., the passive dissipation of task-sets) have been demonstrated to contribute to mixing and switch costs (cf. Monsell, 2003), but the focus of the present investigation is specifically on age differences in executive aspects of advance preparation for an upcoming task-switch.

Previous studies suggest that older adults are particularly susceptible to interference, in particular when task requirements are demanding (e.g., Nessler et al., 2007; Friedman et al., 2009). During the task-switch paradigm, response conflict is pronounced in older adults when stimuli are associated with opposite responses in each task (Mayr, 2001; Meiran et al., 2001). For example, in the paradigm used here, the stimulus " 1 " is associated with the same response irrespective of the currently relevant task-set (congruent stimulus). On the other hand, the correct response for the stimulus " 3 " depends on whether the currently relevant task requires categorizing the target according to which digit or how many digits are displayed (incongruent stimulus). The performance difference for incongruent relative to congruent stimuli is referred to as the congruency effect (Kiesel et al., 2010). Selecting the correct response on incongruent trials depends on the implementation of the currently relevant task rule, which appears to be very difficult for older adults. Other evidence shows that older adults tend to continue to observe visually presented cues that no longer hold task-relevant information (Spieler et al., 2006), suggesting that older adults have difficulties in maintaining an active representation of the relevant task rule. Finally, mixing costs (reflecting task-set updating and rule representation) are typically pronounced in older adults, whereas switch costs (reflecting taskset reconfiguration) are often not reliably larger in older compared to younger adults (e.g., Kray and Lindenberger, 2000; Meiran et al., 2001; Monsell, 2003). Notably, Mayr (2001) reported age-related differences in behavioral mixing costs for tasks with overlapping stimulus-response rules, but these age differences disappeared when each task was associated with non-overlapping stimulusresponse rules (Mayr, 2001). Taken as a whole, the older-adult impediment with overlapping stimulus-response rules, attention to task-irrelevant cues, and increased congruency effects point to impaired rule representation in this age group (Lien et al., 2008). By contrast, small differences in switch costs between young and older adults suggest that task-set reconfiguration is less affected by aging.

With longer time for advance preparation (i.e., longer CTIs), behavioral mixing costs, associated with task-set coordination, and switching costs, associated with task-set reconfiguration, decrease for both young and older adults. In young adults, mixing and switch costs decrease until the CTI reaches a length of $600 \mathrm{~ms}$
(Monsell, 2003). For older adults, these costs continue to decrease following longer CTIs (between approximately 1000 and $1200 \mathrm{~ms}$ (Cepeda et al., 2001; Meiran et al., 2001). Consequently, additional preparation time beyond $600 \mathrm{~ms}$ reduces age-related differences in behavioral costs, as older adults benefit more than young adults (Cepeda et al., 2001; Meiran et al., 2001). However, the exact mechanisms by which longer preparation contributes to reductions in behavioral costs for young and in particular for older adults are currently unclear.

\section{NEURAL ACTIVITY DURING ADVANCE PREPARATION}

Because a behavioral response is not given during the CTI, the cognitive processes that are responsible for the reduction of behavioral costs in older compared to young adults following long preparatory intervals need to be inferred by the measurement of neural activity (Karayanidis et al., 2010). To this end, two methods have been applied in previous studies: functional magnetic resonance imaging (fMRI) and event-related potentials (ERPs). In a number of recent fMRI studies, hemodynamic activity has been observed in extensive fronto-parietal networks following cue and target presentation in young adults (e.g., Brass and von Cramon, 2002; Braver et al., 2003; Crone et al., 2006a,b; Slagter et al., 2006; Ruge et al., 2009). Specifically, for young adults, rule representation (as reflected by the difference between no-switch and homogeneous trials) has been associated with sustained left-lateral PFC activation (Crone et al., 2006b), whereas task-set reconfiguration (as reflected by the difference between switch and no-switch trials) resulted in medial PFC activity (Crone et al., 2006b; Slagter et al., 2006). Similarly, DiGirolamo et al. (2001) reported greater activation in dorsolateral as well as medial PFC during mixed compared to homogeneous blocks in young adults. By contrast, older adults activated these areas to similar extents for both types of blocks (DiGirolamo et al., 2001). Although these latter data were collected in a blocked fMRI design and, hence, could not differentiate between switch and no-switch trials during mixed blocks, the pattern of results for young and older adults implies an age difference in the recruitment of frontal areas presumably related to rule representation. Specifically, the extant evidence suggest that older adults recruit PFC areas implicated in rule representation in young adults less selectively (i.e., evidence for overactivation, see, e.g., Reuter-Lorenz and Lustig, 2005, for a review).

Previous ERP task-switching investigations have mainly focused on task-set updating and task-set reconfiguration. In these investigations, a centro-parietally distributed cue-locked P300 component has been observed with onset latencies about 200300 ms (e.g., Kray et al., 2005; Nicholson et al., 2005; Eppinger et al., 2007; Jost et al., 2008; West and Travers, 2008; see Karayanidis et al., 2010, for a recent review). Consistent with the notion that the P300 reflects processes related to task-set updating, its magnitude has consistently shown increases on no-switch relative to homogeneous trials (e.g., Kieffaber and Hetrick, 2005; Jost et al., 2008; Wylie et al., 2009). Older compared to younger adults typically show similar cue-locked P300 amplitudes on no-switch compared to homogeneous trials, but with slightly delayed (peak) latencies. This finding suggests largely intact, but somewhat delayed task-set updating processes (Kray et al., 2005; Eppinger et al., 2007; West and Travers, 2008). 
Consistent with task-specific preparation for an upcoming task-switch, the centro-parietal positivity is still larger for switch trials in young adults with a somewhat more variable onset latency of 300-500 ms (e.g., Kieffaber and Hetrick, 2005; Nicholson et al., 2005; Jost et al., 2008; Karayanidis et al., 2009). This socalled differential switch positivity (Karayanidis et al., 2010) has been associated with the processes comprising task-set reconfiguration. More recent evidence suggests that it consists of several underlying ERP sub-components, including task-specific as well as non-specific preparation (Karayanidis et al., 2010). So far, age differences in task-set reconfiguration have not been examined extensively (e.g., Kray et al., 2005; Eppinger et al., 2007; West and Travers, 2008). Even following relatively long CTIs (1500-2500 ms), peak amplitudes of the differential switch positivity at parietal electrode sites were delayed in older compared to young adults. However, consistent with only small age-related changes in behavioral switch costs, neural activity reflecting task-set reconfiguration was otherwise similar for young and older adults.

Neural correlates of rule representation in young and older adults have not been routinely examined in previous ERP studies, unlike fMRI investigations. This apparent discrepancy between fMRI and ERP measures with respect to frontal cortex activation during paradigms which require the representation of the currently relevant procedural rules may reflect the vast differences in time scale between the two techniques (see also Ruge and Braver, 2007). As scalp-recorded ERP activity reflects voltage changes occurring in the millisecond time range, it can track cognitive processes prompted by task-relevant cues on the time scale with which they unfold. Hence, in the present study ERP activity was used to obtain temporally precise measures of potential age-related differences in task-set coordination (comprising rule representation and task-set updating) and task-set reconfiguration occurring in the cue-target interval.

The goal of the present study was to further characterize age-related differences in advance preparation. Following short preparation, pronounced behavioral costs were predicted for older adults, in particular for incongruent trials, reflecting incomplete, or inefficient advance preparation. Hence, following longer preparation, behavioral benefits were predicted for older, but not young adults. Centro-parietal ERP correlates of task-set updating and task-set reconfiguration were expected for both age groups, possibly with a delayed onset in older adults. In line with previous ERP studies, positive modulations for no-switch relative to homogeneous and for switch relative to no-switch trials between 300 and $600 \mathrm{~ms}$. In line with the notion that older adults may complete advance preparation when given additional time, these ERP correlates were expected to extend beyond $600 \mathrm{~ms}$ for the longer CTI for older adults. A second goal was to examine whether evidence for rule representation could also be detected using ERPs. As previous $\mathrm{fMRI}$ results have associated PFC activations on noswitch compared to homogeneous trials with rule representation in young adults (Crone et al., 2006b; Slagter et al., 2006), a putative ERP correlate of rule representation should be evident over frontal scalp sites. Based on the notion that the PFC exerts control over more posterior brain regions, an early on setting, sustained modulation was expected for no-switch relative to homogeneous trials.

\section{MATERIALS AND METHODS \\ PARTICIPANTS}

Twenty young ( 16 females, mean age 23.3 years, range $20-27$ ) and 20 older adults ( 13 females, mean age 72.5 , range 61-83) participated in the current study ${ }^{1}$. Advertisements were placed in local newspapers and on the internet. All participants were righthanded, native English speakers who had normal or correctedto-normal vision. Participants reported themselves to be in good physical and mental health and free from medications known to affect the central nervous system. The study was approved by the New York State Psychiatric Institute's Review Board. All participants signed informed consent and were paid for their participation.

\section{SCREENING PROCEDURES}

Prior to EEG recording, all participants were screened with neuropsychological tests. A summary of the demographic information and neuropsychological test results can be found in Table 1 (except for age, there were no other reliable between-group differences, $p s>0.25)$. All participants were of average or above average intelligence and achieved a score of 50 or better (out of 57) on the modified mini-mental status (mMMS) examination (Mayeux et al., 1981). Older participants also passed a complete medical and neurological examination administered by a board-certified neurologist, were free from dementia or depression and not limited in the activities of daily living as assessed by a semi-structured interview, the SHORT-CARE (Gurland et al., 1984).

\section{MATERIALS AND PROCEDURE}

The cued task-switching paradigm was based on a task described by Cepeda et al. (2001), with modifications for ERP recording (cf. Czernochowski et al., 2010). Participants responded to one of four stimuli $(1,3,111,333)$, based on one of two tasks: which digit or how many digits were presented. While the stimuli 1 and 333 required the same response regardless of task (congruent trials), displays of 3 and 111 required opposite responses for the two tasks (incongruent trials). Both tasks were presented in homogeneous and mixed blocks, the latter of which required a task-switch after 0,1 , or 2 no-switch trials; switch and no-switch trials were equiprobable. One of two verbal cues, the words "What number?" or "How many?", was presented for $300 \mathrm{~ms}$, followed by a fixation cross which appeared for 300 or $900 \mathrm{~ms}$, resulting in cue-target intervals (CTI) of 600 or $1200 \mathrm{~ms}$. Each participant performed in homogeneous and mixed blocks with short and long CTIs. Short and long CTIs were blocked and separated by a short break. For each CTI, participants first completed two homogeneous blocks of each task with 36 trials, followed by three mixed blocks with

\footnotetext{
${ }^{1}$ A portion of the behavioral results as well as the RT-locked waveforms for young and older adults in the short CTI as a function of RT speed have been reported previously (Czernochowski et al., 2010). The behavioral data are included here to allow a comparison of switch and mixing costs in the short and long CTIs regardless of RT speed. Note, however, that two older participants were replaced relative to the previous older-adult sample due to a large number of blink artifacts during the CTI. Including these two participants did not change the pattern of results observed in the previous paper. The sample of young adults was also included in another recent paper, where developmental differences during task-switching were compared between children, adolescents, and young adults (Manzi et al., 2011).
} 
Table 1 | Demographic and neuropsychological test measures for young and older adults.

\begin{tabular}{|c|c|c|c|c|}
\hline & \multicolumn{2}{|c|}{ Young $(n=20)$} & \multicolumn{2}{|c|}{ Old $(n=20)$} \\
\hline & Mean & SD & Mean & SD \\
\hline Age (years) & 23.32 & 2.03 & 72.50 & 6.66 \\
\hline Education (years) & 15.85 & 1.31 & 16.40 & 2.72 \\
\hline Modified MMS & 54.80 & 2.59 & 55.15 & 1.57 \\
\hline Digits forward & 7.45 & 1.36 & 7.50 & 1.10 \\
\hline Digits backward & 5.65 & 1.31 & 6.10 & 1.47 \\
\hline WAIS-III verbal IQ & 130.75 & 14.53 & $129.93^{1}$ & 16.29 \\
\hline WAIS-III performance IQ & 114.50 & 14.50 & $120.86^{1}$ & 16.97 \\
\hline Depression (SHORT-CARE) & & & $1.29^{1}$ & 1.86 \\
\hline Dementia (SHORT-CARE) & & & $0.07^{1}$ & 0.27 \\
\hline
\end{tabular}

${ }^{1} n=14$. Modified MMS: modified mini-mental status (Mayeux et al., 1981) with a maximum score of 57. WAIS-III: Wechsler Intelligence Scale III. SHORT-CARE (Gurland et al., 1984); cutoff for depression is 6 and for dementia is 7.

84 trials each. Finally, two additional homogeneous blocks were presented.

The target stimulus was displayed until a response was made via button press with the left or right index finger. The next cue was presented after a constant $1000 \mathrm{~ms}$ response-cue interval, during which the fixation cross re-appeared. To increase the discriminability of the two cues, one appeared on a blue, the other on a yellow background. The assignment of task and cue color, the task that was performed first, the order of short and long CTI as well as response hand corresponding to a " 1 " or " 3 " button press were counterbalanced across participants. Participants were asked to respond emphasizing speed and accuracy equally. Prior to the actual experiment, two homogeneous blocks and one mixed block were included as practice to ensure that subjects understood the instructions and were performing adequately.

\section{EEG RECORDING}

EEG activity was recorded from 62 scalp sites placed according to the extended 10-20 system with sintered $\mathrm{Ag} / \mathrm{AgCl}$ electrodes using a ground on the right forehead. Vertical and horizontal electrooculograms were recorded from electrodes placed, respectively, above and below the left eye and at the outer canthus of each eye. Electrode impedance was kept below $5 \mathrm{k} \Omega$. The activity of all scalp electrodes was initially referenced to the nose tip and re-referenced offline to averaged mastoids. EEG and EOG were recorded continuously with Synamp amplifiers (DC; $100 \mathrm{~Hz}$ high-frequency cutoff; $500 \mathrm{~Hz}$ digitization rate).

\section{BEHAVIORAL DATA ANALYSIS}

As age-related performance differences are often observed selectively for more difficult task conditions, congruent and incongruent trials were evaluated separately. For each CTI, the analysis of behavioral results focused on 12 conditions: in homogeneous blocks there were congruent and incongruent trials; in mixed blocks there were congruent and incongruent no-switch and switch trials (see Table 2). Mean RTs and SDs were computed for each participant, and all trials exceeding 2.5 SDs from the individual mean for each of the 12 conditions were excluded from further analysis $(2.9 \%$ of all trials for young adults, and $2.8 \%$ for older adults). This procedure was used to eliminate exceedingly long RTs, as a time limit for responding was not imposed.

Reaction times and percentage of errors were assessed in mixedmodel ANOVAs with the factors Age Group (Young, Old), CTI (600, $1200 \mathrm{~ms}$ ), Congruency (congruent, incongruent), and Trial Type. The factor of Trial Type was defined as homogeneous vs. no-switch trials to examine mixing costs and no-switch vs. switch trials to examine switch costs. Interactions were followed up with subsidiary ANOVAs. In addition, mixing and switch costs were compared to 0 using one-sample $t$-tests.

\section{ERP DATA ANALYSIS}

EEG epochs extended from $100 \mathrm{~ms}$ prior to the cue until target onset. The pre-cue interval served as baseline. Prior to averaging, eye movements were corrected (Gratton et al., 1983) and trials with visible artifacts (e.g., muscular activity) were rejected. If single channels showed artifacts, a spherical spline algorithm (Perrin et al., 1989) was used for interpolation on a trial-by-trial basis, with a maximum of four channels interpolated for a given trial. A $30-\mathrm{Hz}$ low-pass filter was applied to the averages after statistical processing and is reflected solely in the figures.

As congruent and incongruent trials could only be differentiated at target onset (i.e., after the end of the EEG epochs used in the present investigation), ERP averages were constructed for each participant for a total of six conditions: homogeneous, no-switch, and switch trials for the short and long CTIs. The mean trial numbers (range 21-141) for young and older adults in these conditions were: homogeneous trials (short CTI: 133 vs. 113, long CTI: 128 vs. 104), no-switch trials (short CTI: 114 vs. 97, long CTI: 113 vs. 101) and switch trials (short CTI: 110 vs. 95, long CTI: 108 vs. 98).

Consistent with the behavioral analyses, for all ERP analyses the factor of Trial Type was defined as homogeneous vs. noswitch trials to examine mixing costs and no-switch vs. switch trials to examine switch costs. ERP analyses were conducted at two regions of interest (ROIs), comprising four selected centro-parietal (CPZ, P3, PZ, and P4) as well as four antero-frontal electrode sites (AF3, AFZ, AF4, FZ). To examine whether the previously established parietal ERP correlates of task-set updating and task-set reconfiguration could be dissociated from the proposed frontally distributed electrophysiological correlate of rule representation, potential interactions of Trial Type and Anterior-Posterior ROI were evaluated. As previous findings have implicated age differences in particular following short preparation time, potential interactions were evaluated separately for each CTI. For these ANOVAs with the factors Trial Type, ROI (antero-frontal, parietal), and Age Group (young, older adults), a time window of 300-600 ms was selected.

Parietal amplitude differences between no-switch and homogeneous trials were taken to reflect task-set updating, whereas parietal amplitude differences between switch and no-switch trials were attributed to task-set reconfiguration. In order to establish whether the processes reflected in task-set updating, task-set reconfiguration, and rule representation were present for both young and older adults, subsequent ERP analyses were conducted 
Table 2 | Mean error rates and reaction times (+SE) following short and long preparation for young and older adults for homogeneous, no-switch, and switch trials under congruent and incongruent conditions.

\begin{tabular}{|c|c|c|c|c|c|c|c|c|}
\hline \multirow[t]{2}{*}{ Group } & \multirow[t]{2}{*}{ CTI } & \multirow[t]{2}{*}{ Congruency } & \multicolumn{3}{|c|}{ Error rates } & \multicolumn{3}{|c|}{ Reaction times } \\
\hline & & & Homogeneous & No-switch & Switch & Homogeneous & No-switch & Switch \\
\hline & & Incongruent & $4.21(0.72)$ & $6.93(1.07)$ & $12.63(1.54)$ & $373(17)$ & $442(31)$ & 469 (31) \\
\hline & Long & Congruent & $1.15(0.25)$ & $0.93(0.27)$ & $1.04(0.23)$ & $366(18)$ & $402(27)$ & $405(28)$ \\
\hline & & Incongruent & $5.30(0.85)$ & $7.73(1.11)$ & $12.82(1.64)$ & $375(21)$ & $443(33)$ & 456 (34) \\
\hline & & Incongruent & $2.52(0.72)$ & $3.93(1.03)$ & $7.06(1.54)$ & $565(17)$ & $670(31)$ & 709 (31) \\
\hline & Long & Congruent & $0.21(0.25)$ & $0.41(0.41)$ & $0.34(0.23)$ & $509(18)$ & $577(27)$ & $587(28)$ \\
\hline & & Incongruent & $1.94(0.85)$ & $3.74(1.11)$ & $7.47(1.6)$ & $539(21)$ & $636(33)$ & $662(34)$ \\
\hline
\end{tabular}

separately for each age group and CTI. Task-set updating and reconfiguration were evaluated in the centro-parietal ROI (CPZ, P3, PZ, and P4). To explicitly examine whether task-set updating and/or reconfiguration are delayed in older relative to young adults, the two sets of processes were evaluated in consecutive 100ms time windows. These analyses were conducted between 100 and $600 \mathrm{~ms}$, and between 100 and $1200 \mathrm{~ms}$ following cue onset for the short and long CTIs, respectively.

To further characterize rule representation, the onset and offset of frontal effects was evaluated in corresponding analyses in the antero-frontal ROI (AF3, AFZ, AF4, FZ), consistent with the functional role of the frontal cortex in rule representation. This analysis also examined mean amplitudes in consecutive 100-ms time windows for both age groups and CTIs. In these analyses, amplitude differences between no-switch and homogeneous trials were expected to reflect rule representation, i.e., the continued active maintenance of the previously relevant stimulus-response rule. No differences were expected between no-switch and switch trials.

An $\alpha$-level of 0.05 was chosen for all analyses. To avoid alpha error accumulation, two further restrictions were imposed for the more detailed analyses in consecutive $100 \mathrm{~ms}$ time windows: as sustained effects were predicted, no single $100 \mathrm{~ms}$ time window was considered reliable (minimum of two consecutive $100 \mathrm{~ms}$ time windows with reliable effects). Moreover, $100 \mathrm{~ms}$ time windows were evaluated consecutively, i.e., whenever a non-reliable effect occurred following a reliable one, consecutive time windows were not further analyzed. Partial eta squared $\left(\eta_{p}^{2}\right)$ is given as an estimate of effect size.

\section{RESULTS - BEHAVIORAL DATA}

An overview of the error rates and RTs for the two age groups can be found in Table 2. In order to compare age-related differences in task-set coordination (as evident in mixing costs), errors, and RTs were analyzed in a mixed-model ANOVA with the between-group factor Age Group (young, old), and the repeated-measures factors of Trial Type (homogeneous, no-switch), CTI (600, $1200 \mathrm{~ms})$, and Congruency (congruent, incongruent). In order to examine agerelated differences in task-set reconfiguration (as evident in switch costs), errors, and RTs were analyzed in a corresponding ANOVA comparing switch and no-switch trials.

\section{TASK-SET COORDINATION - MIXING COSTS Accuracy}

More errors occurred for no-switch than homogeneous trials, as evident in a main effect of Trial type $[F(1,38)=23.56, p<0.0001$, $\left.\eta_{p}^{2}=0.38\right]$. Older relative to young adults committed fewer errors (1.67 vs. $3.55 \%)$, as confirmed by a main effect of Age Group $\left[F(1,38)=8.37, p<0.01, \eta_{p}^{2}=0.18\right]$. However, no age-related differences in accuracy mixing costs were observed [no interaction Age group by Trial type $F(1,38)<1]$. More errors occurred for incongruent relative to congruent trials (4.54 vs. $0.68 \%$ ), as evident in a main effect of Congruency $[F(1,38)=63.91$, $\left.p<0.0001, \eta_{p}^{2}=0.63\right]$. This congruency effect was larger for young compared to older adults (1.17 vs. $0.87 \%)$, as suggested by an interaction of Age Group and Congruency $[F(1,38)=5.49$, $\left.p<0.05, \eta_{p}^{2}=0.13\right]$, and also larger for no-switch compared to homogeneous trials (4.93 vs. $2.78 \%),[F(1,38)=21.81$, $\left.p<0.0001, \eta_{p}^{2}=0.37\right]$. The length of the CTI did not influence accuracy $[F(1,38)<1]$ or interact with any other factor (all ps > 0.14).

One-sample $t$-tests indicated that accuracy mixing costs for both short and long CTIs in both groups were reliably different from 0 for incongruent trials only (one-sample $t$ s, $p s<0.05$ ).

\section{Reaction times}

Reaction times on no-switch trials were slower than those on homogeneous trials $\left[F(1,38)=55.54, p<0.0001, \eta_{p}^{2}=0.59\right]$. Young adults responded faster than older adults [396 vs. $576 \mathrm{~ms}$; $F(1,38)=36.58, p<0.0001]$. Moreover, RT mixing costs tended to be larger for older compared to young adults (198 vs. $163 \mathrm{~ms}$ ), as suggested by the marginally significant interaction between Age Group and Trial type $\left[F(1,38)=3.22, p=0.08, \eta_{p}^{2}=0.08\right]$. RTs were slower for incongruent compared to congruent trials (505 vs. $467 \mathrm{~ms}$ ), as evident in a main effect of congruency $\left[F(1,38)=56.67, p<0.0001, \eta_{p}^{2}=0.60\right]$. These Congruency effects (i.e., $\mathrm{RT}_{\text {incongruent }}-\mathrm{RT}_{\text {congruent }}$ ) were more pronounced in older than young adults (52 vs. $24 \mathrm{~ms}$ ), as indicated by an interaction of Age Group with Congruency $[F(1,38)=7.43$, $\left.p<0.01, \eta_{p}^{2}=0.16\right]$, and also larger for no-switch compared to homogeneous trials (52 vs. $25 \mathrm{~ms}$ ), $[F(1,38)=13.93, p<0.01$, $\left.\eta_{p}^{2}=0.27\right]$. Of particular interest for the current investigation, an interaction between Age group, CTI, and Congruency 
$\left[F(1,38)=4.05, p=0.05, \eta_{p}^{2}=0.10\right]$ confirmed that congruency effects in older, but not young adults were modulated by preparation time. This result was further supported by analyses conducted separately for each age group. As expected, for the young, CTI duration did not influence the magnitude of congruency effects (all Fs $<1$ ). By contrast, older adults benefited from the longer preparation time, in particular for incongruent trials, as reflected by the interaction of CTI and Congruency $[F(1,19)=5.85, p<0.05]$.

Reaction time mixing costs were reliably larger than 0 for both age groups and across conditions (one-sample $t$ s, $p s<0.05$ ).

\section{TASK-SET RECONFIGURATION - SWITCH COSTS \\ Accuracy}

More errors occurred on switch than no-switch trials, as confirmed by a main effect of Trial type $[F(1,38)=39.83, p<0.0001$, $\left.\eta_{p}^{2}=0.51\right]$. The main effect of Age Group $[F(1,38)=8.24$, $p<0.01, \eta_{p}^{2}=0.18$ ] indicated that older relative to young adults committed fewer errors (2.99 vs. 5.52\%). However, age-related differences in switch costs were not found $[F(1,38)=1.95, p>0.17]$. More errors occurred on incongruent compared to congruent trials $(7.79$ vs. $0.73 \%)$, as evident in a main effect of Congruency $\left[F(1,38)=90.02, p<0.0001, \eta_{p}^{2}=0.70\right]$, and these congruency effects were larger for young compared to older adults (9.00 vs. $5.12 \%$ ), as indicated by the interaction of Age Group and Congruency $\left[F(1,38)=6.80, p<0.05, \eta_{p}^{2}=0.15\right]$. Congruency effects were also larger for switch compared to no-switch trials $(9.20$ vs. $4.90 \%)$, as indicated by the interaction of Trial Type and Congruency $\left[F(1,38)=34.71, p<0.0001, \eta_{p}^{2}=0.48\right]$. CTI duration did not influence accuracy $[F(1,38)<1]$ or interact with any other factor (all ps > 0.48).

In both groups accuracy switch costs were reliable for incongruent trials only, for both short and long CTIs (one-sample $t$, ps $<0.05)$.

\section{Reaction time}

Responses on switch trials were slower than those on no-switch trials (537 vs. $522 \mathrm{~ms}$ ), $\left[F(1,38)=15.23, p<0.0001, \eta_{p}^{2}=0.29\right]$. Young responded faster than older adults (429 vs. $630 \mathrm{~ms})$, as indicated by a main effect of Age Group $[F(1,38)=28.44$, $\left.p<0.0001, \eta_{p}^{2}=0.43\right]$, but switch costs were age-invariant [interaction of Age Group and Trial type: $F(1,38)<1$ ]. RTs were slower for incongruent compared to congruent trials (561 vs. $498 \mathrm{~ms}$ ), as evident in a main effect of congruency $\left[F(1,38)=81.92, p<0.0001, \eta_{p}^{2}=0.68\right]$. These congruency effects were more pronounced in older compared to young adults ( 79 vs. $47 \mathrm{~ms}$ ), as indicated by the interaction of Age Group and Congruency $\left[F(1,38)=5.50, p<0.05, \eta_{p}^{2}=0.13\right]$. Congruency effects were also more pronounced in switch compared to no-switch trials (75 vs. $52 \mathrm{~ms}$ ), $[F(1,38)=9.12, p<0.01$, $\left.\eta_{p}^{2}=0.19\right]$. No reliable interactions with CTI were observed (all ps $>0.24)$.

One-sample $t$-tests indicated that RT switch costs were only reliable for some conditions: incongruent trials in the short CTI for young [ $27 \mathrm{~ms}, t(19)=2.78, p<0.05]$ and older adults [ $39 \mathrm{~ms}$, $t(19)=2.19, p<0.05]$ and incongruent trials in the long CTI for older adults [26 ms, $t(19)=3.01, p<0.01]$.

\section{ERP RESULTS}

For each CTI, amplitude differences as a function of Trial Type (noswitch vs. homogeneous and switch vs. no-switch, respectively) were examined between 300 and $600 \mathrm{~ms}$ to dissociate effects at antero-frontal and centro-parietal ROIs. To further characterize the onset and duration of task-set updating, rule representation, and task-set reconfiguration in each age group, subsequent ANOVAs evaluated the precise timing of these processes in more detail, as summarized in Tables 3 and 4 . In all instances, more positive waveforms were observed for no-switch vs. homogeneous and switch vs. no-switch conditions.

\section{EFFECTS OF TASK-SET COORDINATION (NO-SWITCH VS. HOMOGENEOUS TRIALS)}

Comparing ERP differences between no-switch and homogeneous trial types during the short CTI, a reliable main effect of Trial Type $\left[F(1,38)=85.55, p<0.0001, \eta_{p}^{2}=0.69\right]$ and an interaction of Trial Type and Age Group $\left[F(1,38)=6.47, p<0.05, \eta_{p}^{2}=0.15\right]$ were observed, as illustrated in Figure 1. As predicted, an interaction with of Trial Type and Anterior-Posterior ROIs was found $\left[F(1,38)=5.84, p<0.05, \eta_{p}^{2}=0.13\right]$. For the long CTI, a main effect of Trial Type $\left[F(1,38)=62.26, p<0.0001, \eta_{p}^{2}=0.62\right]$ and the predicted interaction of Trial Type and Anterior-Posterior ROIs were observed $\left[F(1,38)=5.24, p<0.05, \eta_{p}^{2}=0.12\right]$, but interactions with Age Group were no longer reliable (all $p s>0.16$ ).

\section{Parietal correlates of task-set coordination (task-set updating)}

To parse these interactions and to further characterize the timing of centro-parietal and antero-frontal effects, subsequent ANOVAs were conducted in consecutive $100 \mathrm{~ms}$ time windows. As illustrated in Figures 2 and 3, for young adults reliable differences between no-switch and homogeneous trials were found between 200 and $600 \mathrm{~ms}$ during both CTIs. For older adults, differences between no-switch and homogeneous trials were found between 100 and $600 \mathrm{~ms}$ during the short CTI (Figure 4). For the long CTI, these differences were sustained from 100 to 1100 ms (Figure 5).

\section{Frontal correlates of task-set coordination (rule representation)}

As predicted, reliable differences between no-switch and homogeneous trials were found for young adults between 100 and $600 \mathrm{~ms}$ during the short CTI, and between 100 and $500 \mathrm{~ms}$ during the long CTI. For older adults, these differences were observed between 200 and $400 \mathrm{~ms}$ during the short CTI (Figure 4). During the long CTI, this positivity was present between 100 and $900 \mathrm{~ms}$ (Figure 5).

In a next step, the proposed functional relevance of frontal ERP effects for rule representation was evaluated. To this end, ERP amplitude differences between no-switch and homogeneous trials (300-600 ms) were correlated with a performance measure of costs associated with inefficient rule representation for this condition, namely congruency costs for no-switch trials: during the short CTI, larger amplitude differences were associated with smaller congruency costs across age groups $(r=-0.46, p<0.01)$ and among older adults only $(r=-0.58, p<0.01)$. For young adults, this association was not as strong and not reliable $(r=-0.25$, $p=0.28)$. During the long CTI, this association was no longer observed, $(r=-0.10, p=0.56$ across age groups and $r=-0.07$, 
Table 3 | Results of the trial type (no-switch, homogeneous) ANOVA for parietal and antero-frontal regions of interest.

\begin{tabular}{|c|c|c|c|c|c|c|c|c|}
\hline Age group & CTI & Time window & $\begin{array}{l}\text { Centro-parietal } \\
\text { ROI } F(1,19)\end{array}$ & $p$ & $\eta_{p}^{2}$ & $\begin{array}{l}\text { Antero-frontal } \\
\mathrm{ROI} F(1,19)\end{array}$ & $p$ & $\eta_{p}^{2}$ \\
\hline \multirow[t]{8}{*}{ Young } & Short & $100-200$ & ns & & & 16.95 & 0.001 & 0.47 \\
\hline & & $300-400$ & 59.53 & $<0.0001$ & 0.76 & 9.17 & 0.007 & 0.33 \\
\hline & & $400-500$ & 65.23 & $<0.0001$ & 0.77 & 33.28 & $<0.0001$ & 0.64 \\
\hline & & $500-600$ & 15.80 & 0.001 & 0.45 & 5.92 & 0.025 & 0.24 \\
\hline & & 200-300 & 9.91 & 0.005 & 0.34 & 10.54 & 0.004 & 0.36 \\
\hline & & $300-400$ & 29.70 & $<0.0001$ & 0.61 & 4.51 & 0.047 & 0.19 \\
\hline & & $400-500$ & 33.47 & $<0.0001$ & 0.64 & 16.44 & 0.001 & 0.46 \\
\hline & & $500-600$ & 10.13 & 0.005 & 0.35 & ns & & \\
\hline \multirow[t]{12}{*}{ Older } & Short & 100-200 & 8.82 & 0.008 & 0.32 & ns & & \\
\hline & & $200-300$ & 18.42 & $<0.0001$ & 0.49 & 24.11 & $<0.0001$ & 0.56 \\
\hline & Long & 100-200 & 14.84 & 0.001 & 0.44 & 13.74 & 0.001 & 0.42 \\
\hline & & $200-300$ & 18.60 & $<0.0001$ & 0.50 & 42.97 & $<0.0001$ & 0.69 \\
\hline & & $300-400$ & 10.56 & 0.004 & 0.36 & 9.27 & 0.007 & 0.33 \\
\hline & & $400-500$ & 16.14 & 0.001 & 0.46 & 11.14 & 0.003 & 0.37 \\
\hline & & $500-600$ & 43.21 & $<0.0001$ & 0.70 & 49.70 & $<0.0001$ & 0.72 \\
\hline & & $600-700$ & 43.28 & $<0.0001$ & 0.70 & 42.64 & $<0.0001$ & 0.69 \\
\hline & & $700-800$ & 51.96 & $<0.0001$ & 0.73 & 9.43 & 0.006 & 0.33 \\
\hline & & $800-900$ & 33.55 & $<0.0001$ & 0.64 & 5.50 & 0.030 & 0.23 \\
\hline & & $900-1000$ & 29.09 & $<0.0001$ & 0.61 & ns & & \\
\hline & & $1000-1100$ & 12.01 & 0.003 & 0.39 & ns & & \\
\hline
\end{tabular}

Trial type F-ratios and partial eta squared $\left(\eta_{p}^{2}\right)$ are tabled for reliable effects in at least two consecutive time windows ( $\left.p<0.05\right)$.

$p=0.76$ for older adults only), consistent with the lack of age interactions for the long CTI described above (see also Figure 1).

To summarize the pattern of effects in each age group, for young adults, sustained effects were observed at both centro-parietal and antero-frontal ROIs. Antero-frontal effects preceded those from parietal electrode sites by $100 \mathrm{~ms}$, and no reliable differences were observed beyond $600 \mathrm{~ms}$ during the long CTI. For older adults, parietal effects were sustained throughout both CTIs (until 600 and $1100 \mathrm{~ms}$, respectively). Antero-frontal effects were delayed by $100 \mathrm{~ms}$ and of shorter duration for the short CTI, but prolonged until 900 ms during the long CTI.

\section{EFFECTS OF TASK-SET RECONFIGURATION (SWITCH VS. NO-SWITCH TRIALS)}

Comparing switch and no-switch trials during the short CTI revealed a main effect of Trial Type $[F(1,38)=19.89, p<0.0001$, $\left.\eta_{p}^{2}=0.34\right]$, as illustrated in Figure 6. As predicted, this effect was modified by an interaction with Anterior-Posterior ROIs $\left[F(1,38)=33.94, p<0.0001, \eta_{p}^{2}=0.47\right]$ and a three-way interaction including Age Group $\left[F(1,19)=22.09, p<0.0001, \eta_{p}^{2}=\right.$ 0.37]. During the long CTI, the same pattern of results emerged, including a main effect of Trial Type $[F(1,38)=25.16, p<0.0001$, $\left.\eta_{p}^{2}=0.40\right]$, an interaction of Trial Type and Anterior-Posterior ROIs $\left[F(1,38)=7.48, p<0.01, \eta_{p}^{2}=0.16\right]$ and a three-way interaction with the factor Age Group $[F(1,19)=7.10, p<0.05$, $\left.\eta_{p}^{2}=0.16\right]$.

\section{Parietal correlates of task-set reconfiguration}

As illustrated in Figures $\mathbf{2}$ and 3, for young adults parietal differences between switch and no-switch trials were reliable from 100 to $600 \mathrm{~ms}$ during the short CTI (Table 4). During the long CTI, these parietal effects were sustained until $1100 \mathrm{~ms}$. For older adults, parietal effects were observed between 100 and $600 \mathrm{~ms}$ in the short CTI, and between 300 and $800 \mathrm{~ms}$ during the long CTI (Figures 4 and 5).

\section{Frontal ERP correlates of task-set reconfiguration?}

As predicted, in young adults antero-frontal amplitude differences between switch and no-switch trials were not found in either short or long CTIs (see Figures 2 and 3). By contrast, for older adults reliable differences were observed between 300 and $600 \mathrm{~ms}$ during both CTIs (Figures $\mathbf{4}$ and $\mathbf{5}$ ).

To summarize the pattern of effects in each age group, a reliable parietal positivity was observed throughout the short CTI for young and older adults. During the long CTI, it was sustained until $1100 \mathrm{~ms}$ for young adults. For older adults, it was sustained until $800 \mathrm{~ms}$ with a delayed onset at $300 \mathrm{~ms}$. As predicted, no frontal ERP modulation was observed for young adults, but for older adults between 300 and $600 \mathrm{~ms}$. 
Table 4 | Results of the trial type (switch, no-switch) ANOVA for the parietal and antero-frontal regions of interest.

\begin{tabular}{|c|c|c|c|c|c|c|c|c|}
\hline Group & CTI & Time window & $\begin{array}{l}\text { Centro-parietal } \\
\text { ROI } F(1,19)\end{array}$ & $p$ & $\eta_{p}^{2}$ & $\begin{array}{l}\text { Antero-frontal } \\
\text { ROI } F(1,19)\end{array}$ & $p$ & $\eta_{p}^{2}$ \\
\hline \multirow[t]{9}{*}{ Young } & Short & 100-200 & 10.20 & 0.005 & 0.35 & ns & & \\
\hline & & $300-400$ & 29.72 & $<0.0001$ & 0.61 & ns & & \\
\hline & & $400-500$ & 33.16 & $<0.0001$ & 0.64 & $\mathrm{~ns}$ & & \\
\hline & & $500-600$ & 41.22 & $<0.0001$ & 0.69 & ns & & \\
\hline & & $200-300$ & 4.73 & 0.042 & 0.20 & ns & & \\
\hline & & $300-400$ & 20.03 & $<0.0001$ & 0.51 & ns & & \\
\hline & & $400-500$ & 59.42 & $<0.0001$ & 0.76 & ns & & \\
\hline & & $500-600$ & 41.93 & $<0.0001$ & 0.69 & ns & & \\
\hline & & $600-700$ & 38.77 & $<0.0001$ & 0.67 & ns & & \\
\hline \multirow[t]{10}{*}{ Older } & Short & 100-200 & 7.81 & 0.012 & 0.29 & ns & & \\
\hline & & $200-300$ & 10.49 & 0.004 & 0.36 & ns & & \\
\hline & & $300-400$ & 14.57 & 0.001 & 0.43 & 4.42 & 0.049 & 0.19 \\
\hline & & $400-500$ & 14.76 & 0.001 & 0.44 & 6.75 & 0.018 & 0.26 \\
\hline & & $500-600$ & 12.88 & 0.002 & 0.40 & 8.49 & 0.009 & 0.31 \\
\hline & Long & $300-400$ & 10.78 & 0.004 & 0.36 & 4.50 & 0.047 & 0.19 \\
\hline & & $400-500$ & 10.24 & 0.005 & 0.35 & 5.42 & 0.031 & 0.22 \\
\hline & & $500-600$ & 11.86 & 0.003 & 0.38 & 5.15 & 0.035 & 0.21 \\
\hline & & $600-700$ & 13.13 & 0.002 & 0.41 & ns & & \\
\hline & & $700-800$ & 6.78 & 0.017 & 0.26 & ns & & \\
\hline
\end{tabular}

\section{DISCUSSION}

In the present study, three cognitive processes comprising advance preparation - rule representation, task-set updating, and task-set reconfiguration - were compared between young and older adults. Specifically, the mechanisms contributing to age-related increases in behavioral costs following short preparation and how additional time to prepare may reduce these costs in older adults were evaluated. To summarize the results, RT mixing costs were slightly larger for older compared to young adults. As predicted, older adults were able to take advantage of longer time to prepare, in particular for incongruent trials. Consistent with these behavioral benefits, ERP analyses suggest that age differences during task-set coordination were present for the short, but not the long CTI. Specifically, behavioral interference costs were associated with smaller frontal ERP correlates of rule representation. More detailed analyses in consecutive $100 \mathrm{~ms}$ time windows provide evidence that both rule representation and task-set updating were sustained well into the long CTI for older, but not young adults. Thus, prolonged processing beyond $600 \mathrm{~ms}$ (i.e., the time available during the short CTI) was observed along with performance benefits in older adults. By contrast, behavioral switch costs were small and age-invariant, but ERPs revealed that age differences in task-set reconfiguration were not mitigated by a longer CTI. More detailed analyses suggest that centro-parietal differences between switch and no-switch trials in older adults were smaller in magnitude relative to the young, and also delayed in onset and of shorter duration for the long CTI. In

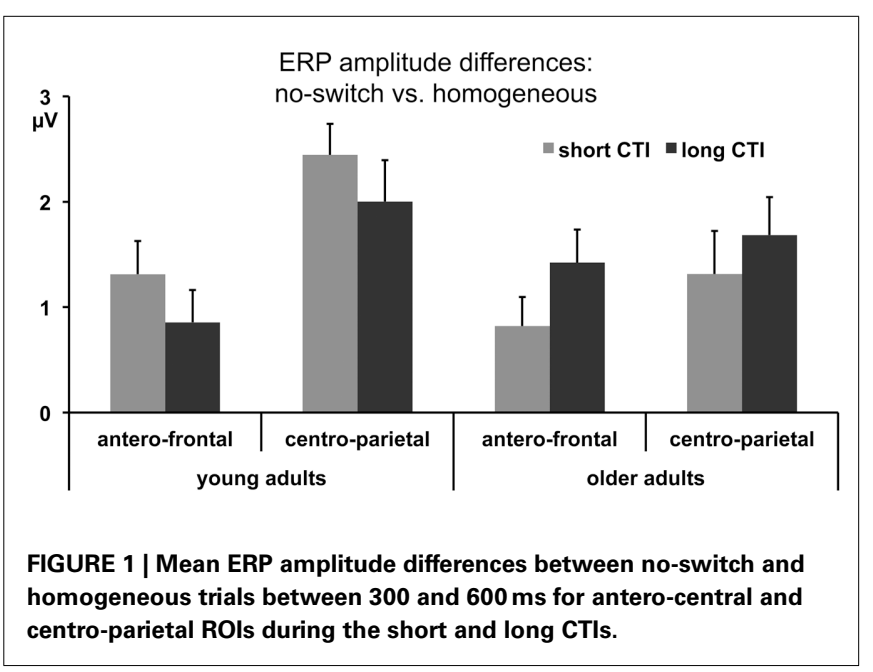

particular, for older but not young adults, antero-frontal amplitude differences between switch and no-switch trials (similar to the ones observed for no-switch compared to homogeneous trials) were observed, in line with less selective PFC activity. As will be discussed in detail below, these behavioral and ERP results suggest an age-related deficit in rule representation during the short CTI, which older adults can overcome when additional time to prepare is available. 


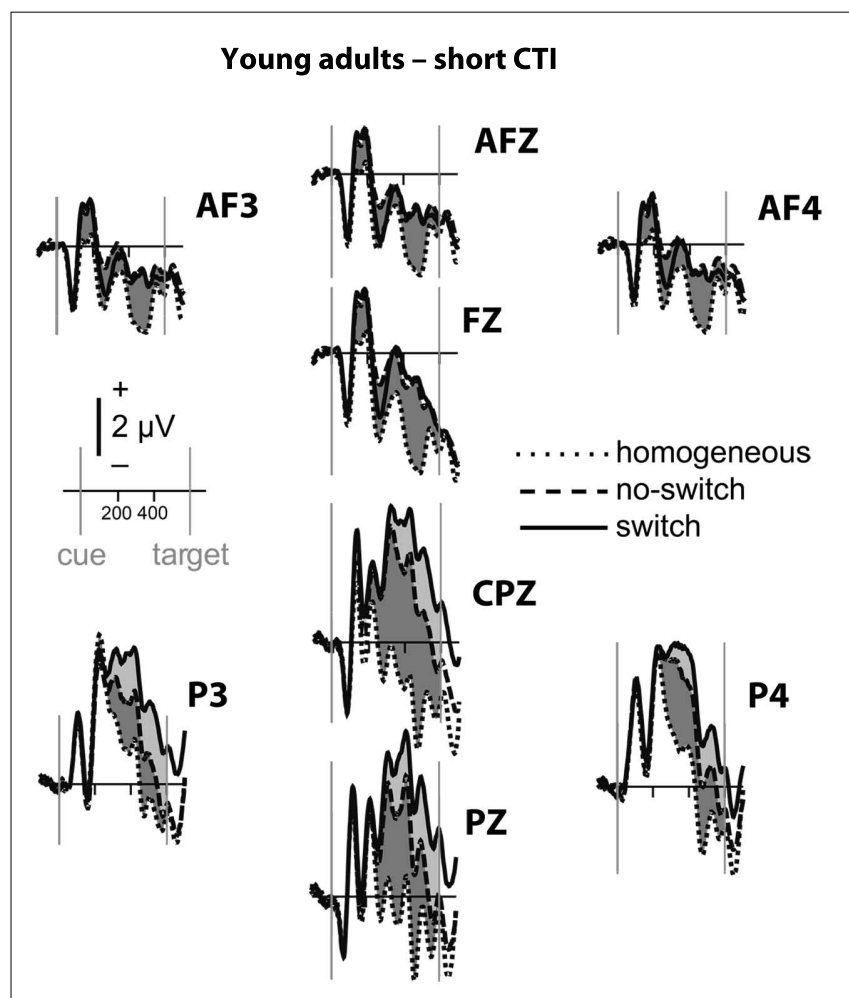

FIGURE 2 | Event-related potential waveforms for young adults during the short CTI. Homogeneous trials (plotted in dotted lines), no-switch trials (in dashed lines), and switch trials (solid lines). Arrows mark cue and target onsets, with time lines every $200 \mathrm{~ms}$. Reliable differences between no-switch and homogeneous trials in the parietal (P3, PZ, P4, CPZ) and antero-frontal (AF3, AFZ, AF4, FZ) region of interest are shaded in light gray, and between switch and no-switch trials in dark gray.

The behavioral results of the present investigation replicate and extend previous findings (e.g., Cepeda et al., 2001; Meiran et al., 2001). Consistent with previous findings, both groups showed reliable RT mixing costs, which were slightly larger in older adults. Notably, older adults were disproportionately slower to respond on incongruent compared to congruent trials (e.g., Mayr, 2001; Meiran et al., 2001). As incongruent trials in particular require the active maintenance of the currently relevant task rule, this pattern of behavioral difficulties is consistent with a specific agerelated deficit in rule representation. Additional time to prepare had no effects on young adults, but was associated with a specific reduction of RTs on incongruent trials in older adults. Typically, in young adults mixing and switch costs decrease with additional time to prepare, but congruency effects are not influenced by additional time (see Kiesel et al., 2010, for a review). However, a plateau in performance improvements is usually reached with CTIs about $600 \mathrm{~ms}$, suggesting that preparation is complete for young adults by that time (see Monsell, 2003, for a review). As predicted, increasing preparation time beyond $600 \mathrm{~ms}$ did not influence overall performance in young adults. Likewise, error rates and RT switch costs in older adults were unaffected by preparation time, suggesting that the small costs associated with task-set reconfiguration are not a result of incomplete preparation in older adults, either. Similarly,

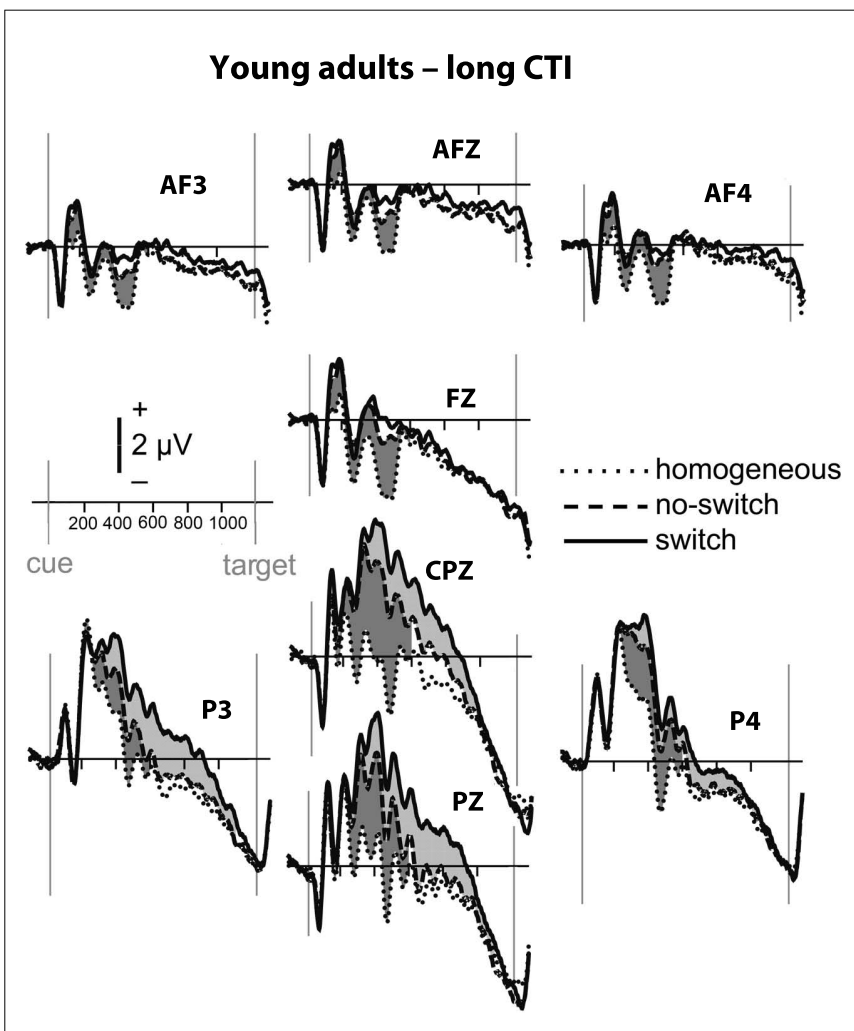

FIGURE 3 | Event-related potential waveforms for young adults during the long CTI. Please refer to Figure 2 for details.

in the present investigation additional time to prepare did not further reduce mixing or switch costs in older adults, which were not particularly pronounced here.

Compared with previous studies, in the present investigation a specific age-related difficulty with incongruent trials was successfully ameliorated by using two relatively long CTIs (600 vs. $1200 \mathrm{~ms}$ ). Beside the length of these CTIs and a relatively long response-cue interval of $1000 \mathrm{~ms}$, several other methodological details might have been critical to obtain these results. First, frequent task-switches were required in the paradigm adopted here, thus encouraging advance preparation. Second, meaningful verbal cues were used in this paradigm to specify each task, which minimized the need to retrieve arbitrary cue-task associations from long-term memory, a task in which age deficits have been shown previously (e.g., Naveh-Benjamin, 2000). Moreover, as task instructions to verbalize each task following cue onset have been shown to improve task-switching performance in older adults (Kray et al., 2008), the use of explicit verbal cues might have had a similar effect, at least on some older adults. Third, the cue was only visible for $300 \mathrm{~ms}$ during the CTI. By contrast to many previous studies, advance preparation was thus not only feasible, but also necessary for successful task performance. In line with the present results, short cue durations are typically associated with minimal switch costs (Kiesel et al., 2010), suggesting that longer cue presentations are not necessarily beneficial. This pattern of results is in line with the notion that older adults tend to observe irrelevant 


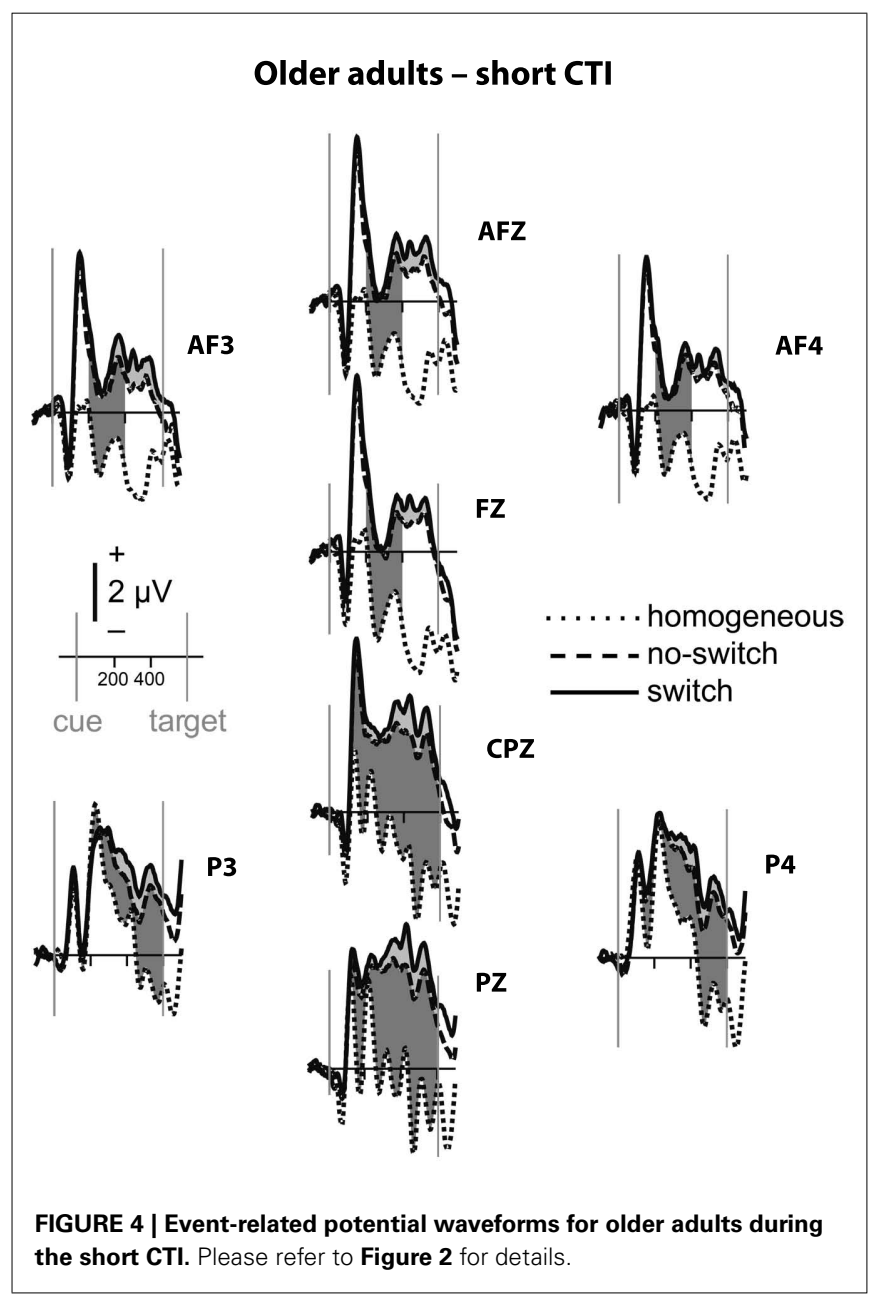

task cues (Spieler et al., 2006). Finally, the length of CTI did not vary randomly within a block (e.g., Meiran et al., 2001; see also Wylie et al., 2009, for a discussion), but was held constant for several blocks. This predictable additional opportunity for advance preparation might have encouraged and/or enabled older adults to prepare more efficiently for the upcoming task. To find out how older adults used the additional time and which mechanisms were responsible for the observed age differences, ERP correlates of task-set updating, task-set reconfiguration, and rule representation were compared between the age groups and will be discussed in turn below.

Task-set updating is thought necessary to determine which task-set is currently relevant. In line with previous ERP studies (Kray et al., 2005; Eppinger et al., 2007; Jost et al., 2008; West and Travers, 2008), cues signaling a no-switch trial in mixed blocks elicited a reliable P300 component over parietal electrodes relative to cues preceding homogeneous trials. These ERP correlates of task-set updating started by $\sim 200 \mathrm{~ms}$ following cue presentation and were sustained for the entire short CTI. Consistent with the lack of behavioral differences between the CTIs for young adults, the ERP correlate of task-set updating was not sustained beyond $600 \mathrm{~ms}$, indicating that young adults were fully prepared by that time. By contrast, for older adults, ERP correlates of task-set

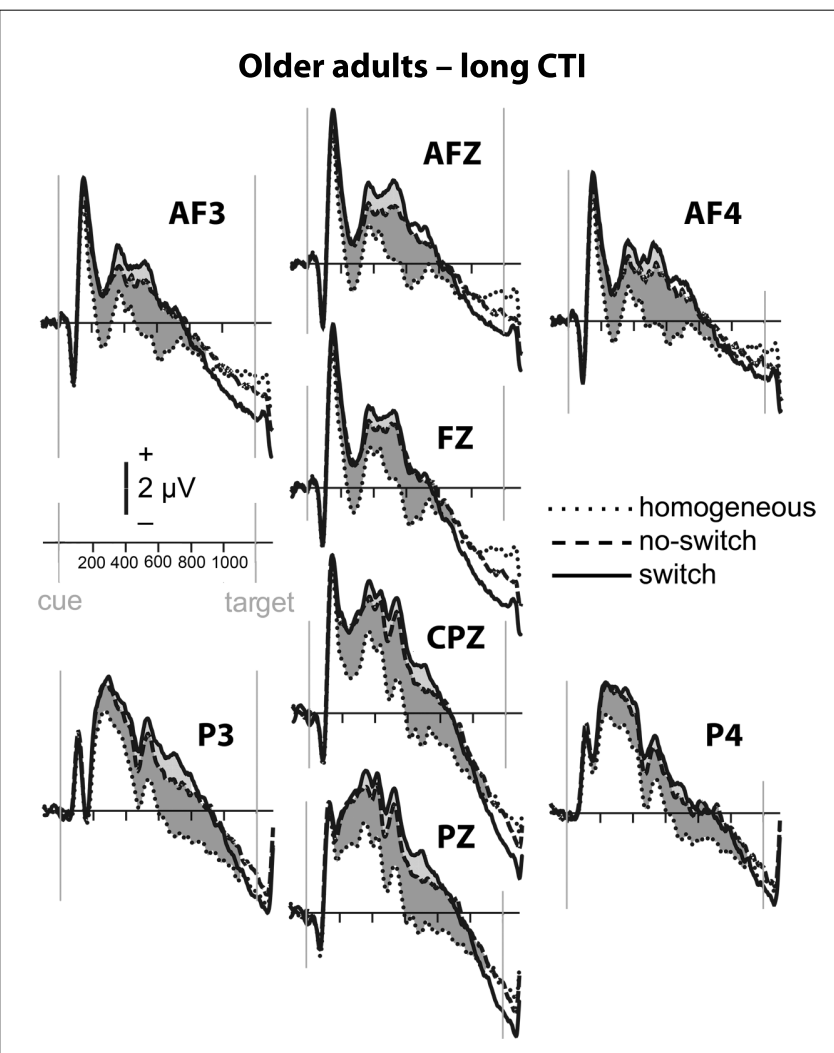

FIGURE 5 | Event-related potential waveforms for older adults during the long CTI. Please refer to Figure 2 for details.

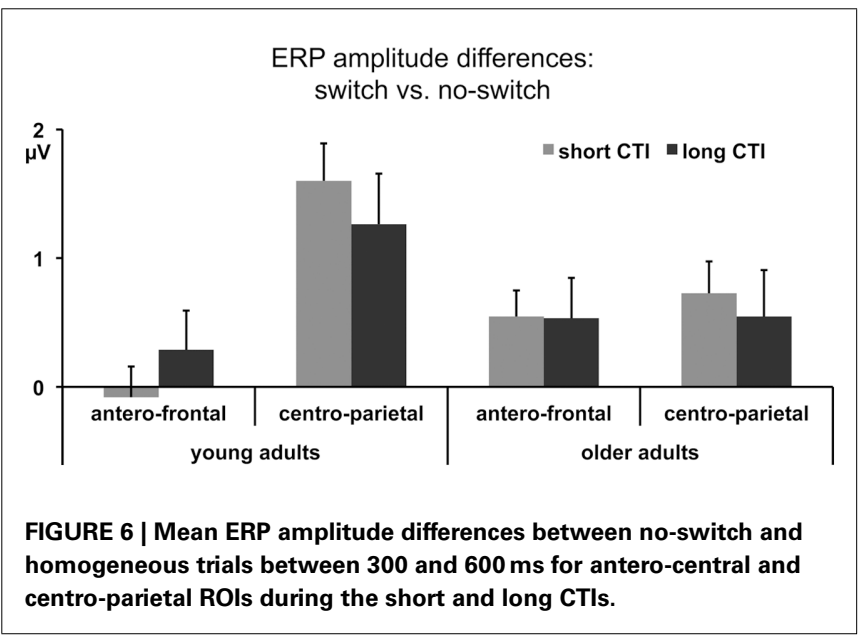

updating were observed as early as $100 \mathrm{~ms}$ following cue presentation, suggesting task-set updating was not delayed, but actually observed earlier in older adults. Between 300 and $600 \mathrm{~ms}$ (i.e., following cue offset), differences between no-switch and homogeneous trials were smaller for older adults during the short CTI, but these age differences disappeared in the long CTI. Moreover, the ERP correlate of task-set updating was sustained for twice as long in older compared to young adults. Together, this pattern of results suggests that older adults had difficulty during the later 
stages of task-set updating for the short CTI (see also Oberauer et al., 2003). Since the cue was no longer visible during this time, older adults might have had difficulties with maintaining an active representation of relevant information without relying on external cues (Spieler et al., 2006). Although the cue was not presented longer during the long CTI, additional, and sustained parietal ERP correlates of task-set updating were observed, indicating that older adults took advantage of the additional time to prepare. Together with the reduced behavioral costs for the long CTI, this additional brain activity is consistent with successful compensation, as will be discussed below in more detail. Compared with previous studies, the present analyses allow a more precise description of the onset and duration of the ERP correlate of task-set updating in older adults. Complementing previous analyses reporting delayed peak latencies for older compared to young adults (Kray et al., 2005; Eppinger et al., 2007), the present results based on mean ERP amplitudes suggest that the ERP correlates of task-set updating were not delayed, but rather smaller in size during the short CTI. Moreover, to the best of my knowledge, the sustained ERP differences between homogeneous and no-switch trials provide the first evidence that older adults used the entire long CTI for task-set updating.

With respect to task-set reconfiguration, for young adults parietal ERP differences between switch and no-switch trials were observed between 100 and $600 \mathrm{~ms}$ during the short CTI, in line with previous findings (e.g., Nicholson et al., 2005; Jost et al., 2008). Despite equivalent performance for both CTIs, in young adults ERP correlates of task-set reconfiguration were sustained until 1100 ms during the long CTI. Together, this pattern of results suggests that young adults continued to process cues indicating an upcoming switch trial when time was available, even if this additional processing did not directly translate into a further reduction of behavioral switch costs. Considerable age-related differences were observed during task-set reconfiguration. For older adults, ERP correlates of task-set reconfiguration in the short CTI were smaller in magnitude from 300 to $600 \mathrm{~ms}$ compared to the young. Notably, these age differences during the processing of cues signaling a task-switch were still evident when additional time to prepare was available during the long CTI: ERP correlates of task-set reconfiguration in older adults started $200 \mathrm{~ms}$ later, ended $300 \mathrm{~ms}$ earlier, and were still considerably smaller in magnitude compared to young adults. As detailed above, ERPs revealed age-related differences in task-set reconfiguration despite similar behavioral performance. Several factors might contribute to this apparent discrepancy. ERPs might be a more sensitive indicator for subtle differences in one or several of the cognitive processes comprising advance preparation, which all lead to only one measurable behavioral outcome (Karayanidis et al., 2010). Moreover, age differences observed for the no-switch condition, in particular the sustained task-set updating effect during the long CTI, contribute to smaller effects during task-set reconfiguration, thus underestimating the overall amount of cognitive resources older adults devoted to switch cues. Still, smaller ERP correlates for taskset reconfiguration compared to task-set updating suggest that older adults do not distribute their attentional focus efficiently toward cues indicating a switch, relative to cues indicating to repeat the previously relevant task-set. Finally, the lack of behavioral differences might be related to a concurrent compensatory activity at other electrode sites observed in older, but not young adults, which will be discussed in more detail below. To conclude, agerelated ERP differences during task-set reconfiguration were found despite equivalent performance.

To identify an ERP correlate of rule representation, the pattern of amplitude differences between no-switch and homogeneous trials was dissociated between centro-parietal and antero-frontal scalp sites. Unlike homogeneous trials, no-switch trials require the active maintenance of the previously relevant stimulus-response rule. A stable rule representation should enable participants to promptly select the correct response for both congruent and incongruent trials, as it will be activated prior to potential interference from incongruent target stimuli. Consistent with these task requirements and with previous fMRI findings (see also Bunge, 2004; Crone et al., 2006b), in young adults an antero-frontal ERP modulation was observed for no-switch compared to homogeneous trials. Consistent with its proposed function, this ERP modulation was sustained during the entire short CTI, and between 100 and $500 \mathrm{~ms}$ during the long CTI in young adults. In line with the proposed PFC function of top-down control on more posterior brain regions, its onset was $\sim 100 \mathrm{~ms}$ earlier compared to parietal effects (see also Brass et al., 2005, for a related argument). As no-switch and switch trials require similar amounts of rule representation, antero-frontal differences between these trial types were neither expected nor observed in young adults. Thus, the pattern of sustained antero-frontal amplitude differences for no-switch compared to homogeneous trials, but not for switch compared to no-switch trials is consistent with the proposed functional role for rule representation in young adults.

Of particular interest for the present investigation was the comparison of age differences in rule representation. Older adults' behavioral difficulties with overlapping stimulus-response rules (Mayr, 2001) and increased attention to task-irrelevant cues (Spieler et al., 2006) may be related to deficits in rule representation. Specifically, incongruent trials require a particularly strong and stable representation of the currently relevant task rule (see Munakata, 2001, for a related argument). In line with the behavioral difficulties observed in older adults, reliable ERP differences between no-switch and homogeneous trials were observed only between 200 and $400 \mathrm{~ms}$ in the short CTI. While visual inspection of the ERP waveforms suggests a somewhat smaller ongoing positive modulation for the remainder of the short CTI, it was likely more variable. Notably, across both groups and for the sample of older adults, a larger frontal ERP effect between 300 and $600 \mathrm{~ms}$ was associated with smaller congruency effects for no-switch trials. No association was found for young adults, presumably due to less variability in ERP amplitudes and small congruency costs across young individuals. Likewise, no association between anterofrontal ERP effects and congruency effects was observed during the long CTI, when the frontal ERP modulation was sustained until $900 \mathrm{~ms}$, and smaller behavioral costs were observed for incongruent trials across groups. Together, this pattern of results is consistent with previous results suggesting that older adults have difficulty when holding information in working memory (Oberauer et al., 2003) and maintaining a task context (Braver et al., 2001). In line with this view, older adults would encounter 
difficulty when required to maintain an active representation of the rules used to respond on the previous trial. Hence, they might benefit less from task repetitions than young adults, resulting in larger mixing costs. While young adults are able to maintain relevant task rules active, older adults might re-activate the appropriate rule on no-switch as well as switch trials. Consistent with this idea, the ERP correlate of task-set updating was particularly large for older compared to young adults, suggesting that the cognitive processes recruited on a portion of no-switch trials more closely resembled task-set reconfiguration than task-set updating for older adults. While this strategy appears to offer a viable solution to overcome behavioral interference, it would require extra time, as evident in correct, but slow responses observed for older adults. To summarize, age-related deficits in rule representation were pronounced for the short CTI, leading to increased interference from the currently irrelevant stimulus-response rule and hence to slow responses in particular for incongruent trials, but these deficits were ameliorated during the long CTI.

As no-switch and switch trials require similar amounts of rule representation, antero-frontal differences between these trial types were neither expected nor observed in young adults. By contrast, reliable differences between these conditions were observed after cue offset for older adults, suggesting older adults recruited additional cognitive operations on switch relative to no-switch trials. As both conditions require the same amount of rule representation, the functional relevance of this ERP modulation for switch trials in older adults remains to be investigated in future studies. This additional activation, as evident in the lack of Trial Type interactions with antero-frontal and parietal ROIs, might reflect so-called dedifferentiation, older adults' difficulties to selectively recruit only task-relevant brain areas (e.g., DiGirolamo et al., 2001; see ReuterLorenz and Lustig, 2005, for a review). However, together with

\section{REFERENCES}

Brass, M., Ullsperger, M., Knoesche, T. R., von Cramon, D. Y., and Phillips, N. A. (2005). Who comes first? The role of the prefrontal and parietal cortex in cognitive control. J. Cogn. Neurosci. 17, 1367-1375.

Brass, M., and von Cramon, D. Y. (2002). The role of the frontal cortex in task preparation. Cereb. Cortex 12, 908-914.

Braver, T. S., Barch, D. M., Keys, B. A., Carter, C. S., Cohen, J. D., Kaye, J. A., Janowsky, J. S., Taylor, S. F., Yesavage, J. A., Mumenthaler, M. S., Jagust, W. J., and Reed, B. R. (2001). Context processing in older adults: evidence for a theory relating cognitive control to neurobiology in healthy aging. J. Exp. Psychol. Gen. 130, 746-763.

Braver, T. S., Reynolds, J. R., and Donaldson, D. I. (2003). Neural mechanisms of transient and sustained cognitive control during task switching. Neuron 39, 713-726.

Bunge, S. A. (2004). How we use rules to select actions: a review of evidence from cognitive neuroscience. Cogn. Affect. Behav. Neurosci. 4, 564-579.

Cepeda, N. J., Kramer, A. F., and Gonzalez de Sather, J. C. (2001). Changes in executive control across the life span: examination of task-switching performance. Dev. Psychol. 37, 715-730.

Crone, E. A., Donohue, S. E., Honomichl, R., Wendelken, C., and Bunge, S. A. (2006a). Brain regions mediating flexible rule use during development. J. Neurosci. 26, 11239-11247.

Crone, E. A., Wendelken, C., Donohue, S. E., and Bunge, S. A. (2006b). Neural evidence for dissociable components of task-switching. Cereb. Cortex 16, 475-486.

Czernochowski, D., Fabiani, M., and Friedman, D. (2008). Use it or lose it? SES mitigates age-related decline in a recency/recognition task. Neurobiol. Aging 29, 945-958.

Czernochowski, D., Nessler, D., and Friedman, D. (2010). On why not to rush older adults-relying on reactive cognitive control can effectively

the lack of age-related performance differences for switch costs, this ERP pattern is also consistent with successful compensatory brain activity during the most difficult task condition recruited by older, but not young adults (e.g., Czernochowski et al., 2008; see Reuter-Lorenz and Lustig, 2005, for a review). Thus, the present results indicate that older adults recruit brain areas not activated in the young for switch compared to no-switch trials, although the functional relevance of this frontal overactivation in older adults remains to be investigated.

To conclude, the present results provide evidence that scarce rule representation in older adults is responsible for pronounced congruency costs following short preparation. ERPs suggest agerelated deficits in task-set updating and rule representation, resulting in older adults' difficulties in holding the currently relevant procedural rules active for a sustained period of time. Longer preparation enables older adults to re-activate relevant task rules, as evident in temporally sustained ERP correlates of task-set updating and rule representation. As a result, older adults can reduce their behavioral costs for incongruent trials when additional time is available. Age-invariant switch costs appear related to additional, potentially compensatory frontal activity recruited by older adults to overcome difficulties in task-set reconfiguration.

\section{ACKNOWLEDGMENTS}

The author would like to thank Dr. David Friedman and Dr. Doreen Nessler for many valuable comments and fruitful discussions, and Dr. Kathrin Lange for helpful comments on a previous version of this manuscript. The author thanks Mr. Charles L. Brown III for computer programming and technical assistance, and Ms. Rebecca Edelblum and Mr. Cort Horton for their aid in participant recruitment and data collection, and Dr. Yael M. Cycowicz for contributions to early phases of this work.

reduce errors at the expense of slowed responses. Psychophysiology 47, 637-646.

De Jong, R., Berendsen, E., and Cools, R. (1999). Goal neglect and inhibitory limitations: dissociable causes of interference effects in conflict situations. Acta Psychol. (Amst.) 101, 379-394.

DiGirolamo, G. J., Kramer, A. F., Barad, V., Cepeda, N. J., Weissman, D. H., Milham, M. P., Wszalek, T. M., Cohen, N. J., Banich, M. T., Webb, A., Belopolsky, A. V., and McAuley, E. (2001). General and task-specific frontal lobe recruitment in older adults during executive processes: a fMRI investigation of task-switching. Neuroreport 12, 2065-2071.

Eppinger, B., Kray, J., Mecklinger, A., and John, O. (2007). Age differences in task switching and response monitoring: evidence from ERPs. Biol. Psychol. 75, 52-67.

Friedman, D., Nessler, D., Cycowicz, Y. M., and Horton, C. (2009). Development of and change in cognitive control: a comparison of children, young adults, and older adults. Cogn. Affect. Behav. Neurosci. 9, 91-102.

Gratton, G., Coles, M. G. H., and Donchin, E. (1983). A new method for off-line removal of ocular artifact. Electroencephalogr. Clin. Neurophysiol. 55, 468-484.

Gurland, B., Golden, R. R., Teresi, J. A., and Challop, J. (1984). The shortcare: an efficient instrument for the assessment of depression, dementia and disability. J. Gerontol. 39, 166-169.

Jost, K., Mayr, U., and Rösler, F. (2008). Is task switching nothing but cue priming? Evidence from ERPs. Cogn. Affect. Behav. Neurosci. 8, 74-84.

Karayanidis, F., Jamadar, S., Ruge, H., Phillips, N., Heathcote, A., and Forstmann, B. U. (2010). Advance preparation in task-switching: converging evidence from behavioral, brain activation and model-based approaches. Front. Psychol. 1:25. doi:10.3389/fpsyg.2010.00025 
Karayanidis, F., Mansfield, E. L., Galloway, K. L., Smith, J. L., Provost, A., and Heathcote, A. (2009). Anticipatory reconfiguration elicited by fully and partially informative cues that validly predict a switch in task. Cogn. Affect. Behav. Neurosci. 9, 202-215.

Kieffaber, P. D., and Hetrick, W. P. (2005). Event-related potential correlates of task switching and switch costs. Psychophysiology 42, 56-71.

Kiesel, A., Wendt, M., Jost, K., Steinhauser, M., Falkenstein, M., Philipp, A. M., and Koch, I. (2010). Control and interference in task switching-a review. Psychol. Bull. 136, 849-874.

Koechlin, E., Ody, C., and Kouneiher, F. (2003). The architecture of cognitive control in the human prefrontal cortex. Science 302, 1181-1185.

Kray, J., Eber, J., and Karbach, J. (2008). Verbal self-instructions in task switching: a compensatory tool for action-control deficits in childhood and old age? Dev. Sci. 11, 223-236.

Kray, J., Eppinger, B., and Mecklinger, A. (2005). Age differences in attentional control: an event-related potential approach. Psychophysiology 42, 407-416.

Kray, J., and Lindenberger, U. (2000). Adult age differences in task switching. Psychol. Aging 15, 126-147.

Lenartowicz, A., Escobedo-Quiroz, R., and Cohen, J. D. (2010). Updating of context in working memory: an event-related potential study. Cogn. Affect. Behav. Neurosci. 10, 298-315.

Lien, M. C., Ruthruff, E., and Kuhns, D. (2008). Age-related differences in switching between cognitive tasks: does internal control ability decline with age? Psychol. Aging 23, 330-341.
Manzi, A., Nessler, D., Czernochowski, D., and Friedman, D. (2011). The development of anticipatory cognitive-control processes in task-switching: an ERP study in children, adolescents and young adults. Psychophysiology 48, 1258-1275.

Mayeux, R., Stern, Y., Rosen, J., and Leventhal, J. (1981). Depression, intellectual impairment, and parkinson disease. Neurology 31, 645-650.

Mayr, U. (2001). Age differences in the selection of mental sets: the role of inhibition, stimulus ambiguity, and response-set overlap. Psychol. Aging $16,96-109$.

Meiran, N., Gotler, A., and Perlman, A. (2001). Old age is associated with a pattern of relatively intact and relatively impaired task-set switching abilities. J. Gerontol. B Psychol. Sci. Soc. Sci. 56, P88-P102.

Miller, E. K., and Cohen, J. D. (2001). An integrative theory of prefrontal cortex function. Annu. Rev. Neurosci. 24, 167-202.

Monsell, S. (2003). Task switching. Trends Cogn. Sci. (Regul. Ed.) 7, 134-140.

Munakata, Y. (2001). Graded representations in behavioral dissociations. Trends Cogn. Sci. (Regul. Ed.) 5, 309-315.

Naveh-Benjamin, M. (2000). Adult age differences in memory performance: tests of an associative deficit hypothesis. J. Exp. Psychol. Learn. Mem. Cogn. 26, 1170-1187.

Nessler, D., Friedman, D., Johnson, R. Jr., and Bersick, M. (2007). ERPs suggest that age affects cognitive control but not response conflict detection. Neurobiol. Aging 28, 1769-1782.
Nicholson, R., Karayanidis, F., Poboka D., Heathcote, A., and Michie, P. T. (2005). Electrophysiological correlates of anticipatory taskswitching processes. Psychophysiology 42, 540-554.

Oberauer, K., Wendland, M., and Kliegl, R. (2003). Age differences in working memory - the roles of storage and selective access. Mem. Cognit. 31, 563-569.

O’Reilly, R. C., Noelle, D. C., Braver, T. S., and Cohen, J. D. (2002). Prefrontal cortex and dynamic categorization tasks: representational organization and neuromodulatory control. Cereb. Cortex 12, 246-257.

Perrin, F., Pernier, J., Bertrand, O. and Echallier, J. F. (1989). Spherical splines for scalp potential and current density mapping. Electroencephalogr. Clin. Neurophysiol. 72 , 184-187.

Reuter-Lorenz, P. A., and Lustig, C. (2005). Brain aging: reorganizing discoveries about the aging mind. Curr. Opin. Neurobiol. 15, 245-251.

Ruge, H., and Braver, T. (2007). "Neural mechanisms of cognitive control in cued task-switching: rules, representations, and preparation," in The Neuroscience of Rule-Guided Behavior, eds S. A. Bunge and J. D. Wallis (New York, NY: Oxford University Press), 255-282.

Ruge, H., Braver, T., and Meiran, N. (2009). Attention, intention, and strategy in preparatory control. $\mathrm{Neu}$ ropsychologia $47,1670-1685$.

Slagter, H. A., Weissman, D. H., Giesbrecht, B., Kenemans, J. L., Mangun, G. R., Kok, A., and Woldorff, M. G. (2006). Brain regions activated by endogenous preparatory set shifting as revealed by fMRI. Cogn. Affect. Behav. Neurosci. 6, 175-189.

Spieler, D. H., Mayr, U., and LaGrone, S. (2006). Outsourcing cognitive control to the environment: adult age differences in the use of task cues. Psychon. Bull. Rev. 13, 787-793.

West, R., and Travers, S. (2008). Differential effects of aging on processes underlying task switching. Brain Cogn. 68, 67-80.

Wylie, G. R., Murray, M. M., Javitt, D. C., and Foxe, J. J. (2009). Distinct neurophysiological mechanisms mediate mixing costs and switch costs. J. Cogn. Neurosci. 21, 105-118.

Conflict of Interest Statement: The author declares that the research was conducted in the absence of any commercial or financial relationships that could be construed as a potential conflict of interest.

Received: 31 May 2011; paper pending published: 23 June 2011; accepted: 22 August 2011; published online: 23 September 2011.

Citation: Czernochowski D (2011) ERP evidence for scarce rule representation in older adults following short, but not long preparatory intervals. Front. Psychology 2:221. doi: 10.3389/fpsyg.2011.00221

This article was submitted to Frontiers in Cognition, a specialty of Frontiers in Psychology.

Copyright $\odot 2011$ Czernochowski. This is an open-access article subject to a nonexclusive license between the authors and Frontiers Media SA, which permits use, distribution and reproduction in other forums, provided the original authors and source are credited and other Frontiers conditions are complied with. 